\title{
Upregulated immuno-modulator PD-L1 in malignant peripheral nerve sheath tumors provides a potential biomarker and a therapeutic target
}

\author{
Said Farschtschi ${ }^{1}$ (D) $\cdot$ Lan Kluwe ${ }^{1}$ (D) Su-Jin Park $^{2} \cdot$ Su-Jun $\mathrm{Oh}^{2} \cdot$ Nancy Mah $^{2} \cdot$ Victor-Felix Mautner $^{1} \cdot$ Andreas Kurtz $^{2}$ (D)
}

Received: 13 August 2019 / Accepted: 10 March 2020 / Published online: 19 March 2020

(c) The Author(s) 2020

\begin{abstract}
Background Malignant peripheral nerve sheath tumors (MPNSTs) are rare aggressive sarcomas with poor prognosis. More than half of MPNSTs develop from benign precursor tumors associated with neurofibromatosis type 1 (NF1) which is a tumor suppressor gene disorder. Early detection of malignant transformation in NF1 patients is pivotal to improving survival. The primary aim of this study was to evaluate the role of immuno-modulators as candidate biomarkers of malignant transformation in NF1 patients with plexiform neurofibromas as well as predictors of response to immunotherapeutic approaches. Methods Sera from a total of $125 \mathrm{NF} 1$ patients with quantified internal tumor load were included, and 25 of them had MPNSTs. A total of six immuno-modulatory factors (IGFBP-1, PD-L1, IFN- $\alpha$, GM-CSF, PGE-2, and AXL) were measured in these sera using respective ELISA.

Results NF1 patients with MPNSTs had significantly elevated PD-L1 levels in their sera compared to NF1 patients without MPNSTs. By contrast, AXL concentrations were significantly lower in sera of NF1-MPNST patients. IGFBP-1 and PGE2 serum levels did not differ between the two patient groups. IFN- $\alpha$ and GM-CSF were below the detectable level in most samples.

Conclusion The immuno-modulator PD-L1 is upregulated in MPNST patients and therefore may provide as a potential biomarker of malignant transformation in patients with NF1 and as a response predictor for immunotherapeutic approaches.
\end{abstract}

Keywords Neurofibromatosis type $1 \cdot$ Biomarker $\cdot$ ELISA $\cdot$ Malignant peripheral nerve sheath tumor $\cdot$ PD-L1 $\cdot$ Immunomodulator

\section{Abbreviations}

AXL Soluble tyrosine receptor kinase AXL

FDA Food and Drug Administration of the United

States of America

IGFBP-1 Insulin-like growth factor-binding protein 1

MPNSTs Malignant peripheral nerve sheath tumors

Electronic supplementary material The online version of this article (https://doi.org/10.1007/s00262-020-02548-1) contains supplementary material, which is available to authorized users.

Said Farschtschi

s.farschtschi@uke.de

1 Department of Neurology, University Medical Center Hamburg-Eppendorf, Martinistr. 52, 20246 Hamburg, Germany

2 BIH Center for Regenerative Therapies, Charité University Medicine Berlin, Augustenburger Platz 1, 13353 Berlin, Germany
NF1 Neurofibromatosis type 1

PGE-2 Prostaglandin E2

RANTES Chemokine Regulated upon Activation, Normal T cell Expressed and Secreted, also Chemokine ligand 5 (CCL5)

T2-STIR T2-Short Tau Inversion Recovery

\section{Introduction}

Malignant peripheral nerve sheath tumors (MPNSTs) are aggressive sarcomas with very poor prognosis and limited therapeutic approaches. More than half of the MPNSTs develop from plexiform neurofibromas (PNF) in patients with neurofibromatosis type 1 (NF1), an autosomal-dominant neurocutaneous tumor suppressor gene disorder [1-3]. PNFs themselves are benign tumors and can be found in more than half of NF1 patients. However, PNFs have a high risk of malignant transformation into MPNSTs. 
Consequently, NF1 patients have a high lifetime risk $(8-13 \%)$ of developing MPNSTs, which are the major cause for their reduced life span $[1,2,4,5]$.

Unfortunately, chemotherapy and radiotherapy can hardly improve survival of MPNST patients. So far, complete surgical resection is the solely reliable curative treatment for MPNSTs, which is only possible for cases with timely diagnosis $[2,6]$. Noninvasive biomarkers for early detection of malignant transformation would therefore be valuable.

In a recent study, we found high serum concentrations of two immuno-modulators in NF1 patients with MPNSTs: the insulin-like growth factor-binding protein 1 (IGFBP-1) and the Chemokine Regulated upon Activation, Normal T cell Expressed and Secreted (RANTES) [7]. Moreover, we found an inverse correlation of $\mathrm{CD} 8(+) / \mathrm{CD} 57(+)$ and CD27(-) $\mathrm{T}$ cell fractions with benign internal tumor load [8]. These findings raised the hypothesis of a potential systemic inflammatory response in the tumor microenvironment which may accompany tumor progression and contribute to malignant transformation in NF1 patients. In light of these studies, we analyzed an array of immuno-modulatory proteins, which have been associated with tumor phenotypes and could be exploited for tumor therapy, including IGFBP-1, programmed cell death ligand 1 (PD-L1), the soluble tyrosine receptor kinase AXL, interferon- $\alpha$ (IFN- $\alpha$ ), granulocytemacrophage colony-stimulating factor (GM-CSF), and prostaglandin E2 (PGE2).

Studies reported that binding of PD-L1 to its receptor programmed cell death protein 1 (PD-1) regulate an immune checkpoint, which then suppresses T cell cytotoxicity [9]. Serum PD-L1 is also reported as a biomarker for immune suppression in patients with other tumors [10,11]. Increased PD-1 and PD-L1 expression would provide a rationale to test the efficacy of immune checkpoint inhibitors/immune therapies targeting this pathway $[12,13]$.

AXL is a receptor tyrosine kinase which regulates various vital cellular processes including proliferation, survival, motility, and immunologic response. AXL is overexpressed in several malignancies and was postulated to contribute to oncogenic processes including angiogenesis and therapy resistance to and suppression of immune response. Therefore, AXL is considered as a potential prognostic biomarker for malignancy and as a target for therapy development. AXL has emerged as a key facilitator of immune escape and drug-resistance by cancer cells, leading to aggressive and metastatic cancers $[14,15]$. In a previous study, NF1 patients with PNFs showed increased soluble AXL levels in serum, compared to NF1 patients with only dermal neurofibroma [16].

IFN- $\alpha$ is a possible inhibitor of tumorigenesis as it stimulates the immune response against tumor cells [17], and thus we speculated that it may be downregulated in MPNST patients. GM-CSF plays a critical role in immune modulation and hematopoiesis; however, it is also frequently upregulated in multiple human cancer types, marking these recognizable for the immune system to stimulate dendritic cell (DC) maturation and monocyte/macrophage activity, but it also seems to stimulate tumor growth and metastasis [18]. PGE2 is a hormonal immune regulator, which is also known to suppress T cell receptor signaling [19].

In this background, we design the present study to measure six selected immuno-modulators and potential activators of MPNSTs-tumorigenesis in sera of a total of $125 \mathrm{NF} 1$ patients, among them 25 had MPNSTs: PD-L1, IGFBP-1, AXL, IFN- $\alpha$, GM-CSF, and PGE2.

\section{Materials and methods}

\section{Sample collection}

All the 125 patients were ascertained for the diagnosis of NF1 followed the NIH criteria [20], among them 25 had histologically confirmed MPNSTs. Serum samples from these patients were obtained in the Neurofibromatosis Outpatient Clinic of the Department of Neurology at the University Medical Center Hamburg-Eppendorf, Germany. Serum preparation followed strictly the standardized protocol: Venous blood of up to $20 \mathrm{ml}$ was collected and separated by centrifugation exactly $30 \mathrm{~min}$ later. Serum was then immediately frozen in $100 \mu \mathrm{l}$ aliquots and stored at $-80^{\circ} \mathrm{C}$ until use.

\section{Determination of tumor load}

Whole-body MRI was performed in NF1-patients to quantify total internal tumor volume. MRI and calculation of total tumor volume were performed in analogy to previous studies $[8,21,22]$. All patients underwent an identical scan protocol with the same parameters for slice thickness, gap, orientation, field of view, imaging matrix, image resolution, echo time, repetition time, and inversion time (Siemens Avanto $1.5 \mathrm{~T}$ ). The subjects were imaged in a supine position from head to knee in four steps (head, thorax, abdomen, and legs) in accordance with the maximum range of table movement. Slice thickness is $5-10 \mathrm{~mm}$ without skips between slices. MRI analysis was performed by an experienced radiologist and a physician trained in image analysis of NF1-associated tumors (Said Farschtschi). The analysis was carried out in a blinded manner. Tumor segmentation and volumetry were performed semiautomatically with the heuristics-based software MEDx (V 3.44) using fat-suppressed T2-short tau inversion recovery sequences (T2-STIR). Differences in signal intensity of neurofibroma tissue and surrounding tissue were used to define tumor margins on axial slices. The method used for this automated volumetric analysis 
is sensitive (it detects volume changes as small as 10\%), reproducible (coefficient of variation $0.6 \%-5.6 \%$ ), and produces results similar to manual tumor tracings $(R=0.999)$. Patients were divided in the groups high $(>500 \mathrm{ml})$, medium $(50-300 \mathrm{ml})$ and low/no $\left(<10 \mathrm{~cm}^{3}\right)$ internal tumor.

\section{Enzyme-linked immunosorbent assays (ELISA)}

ELISA were carried out using the protocol of the manufacturer. The used kits were for AXL1, GM-CSF, IFN- $\alpha$, IGFBP-1 (all RayBiotech, ELH, USA), PGE2 (Abnova KA0326, Japan), and PD-L1 (Affymetrix/Thermo Fischer BM2212). Serum concentration of each of these six immuno-modulators was compared between NF1 patients with and without MPNSTs using a $t$ test with two-tailed hypothesis and equal variation.

\section{Statistical analysis}

Statistical analysis was carried out using unpaired $t$ test with a two-tailed hypothesis. Significant level was set at 0.05 .

\section{Results}

PD-L1 was significantly elevated in sera of NF1 patients with MPNSTs compared to NF1 patients without MPNSTs (Fig. 1a). In NF1, the presence of a PNF is a prerequisite to develop an MPNSTs [1]. When we stratified the patient cohorts without MPNSTs but with PNF into groups of $>500 \mathrm{ml}$ and $<300 \mathrm{ml}$ internal PNF tumor load, and those with no internal tumors, additional patterns emerged. PD-L1 was only increased in serum of MPNST patients when compared to patients with PNF, while the PD-L1 levels in NF1 patients without internal tumors was highly variable (Fig. 1b). The distribution is nearly normal.

By contrast, AXL serum concentrations were significantly reduced in MPNST patients compared to patients without MPNSTs (Fig. 2a). When patients without MPNSTs were divided into three groups according to their internal PNF tumor loads, the two groups with PNF loads under $300 \mathrm{ml}$ had significantly lower AXL concentration than patients with high tumor load ( $>500 \mathrm{ml}$ ). By contrast, AXL concentration did not differ significantly between patient without PNF and patient with MPNSTs (Fig. 2b).

IGFBP-1 and PGE2 serum concentrations did not differ between the patients with and without MPNSTs (Figs. 3a, 4a). Interestingly, MPNST patients are clearly divided into two groups regarding IGFBP-1 serum concentration: (1) $50-300 \mathrm{pg} / \mathrm{ml}$ and (2) 600-850 pg/ml (Figs. 3b, 4b).

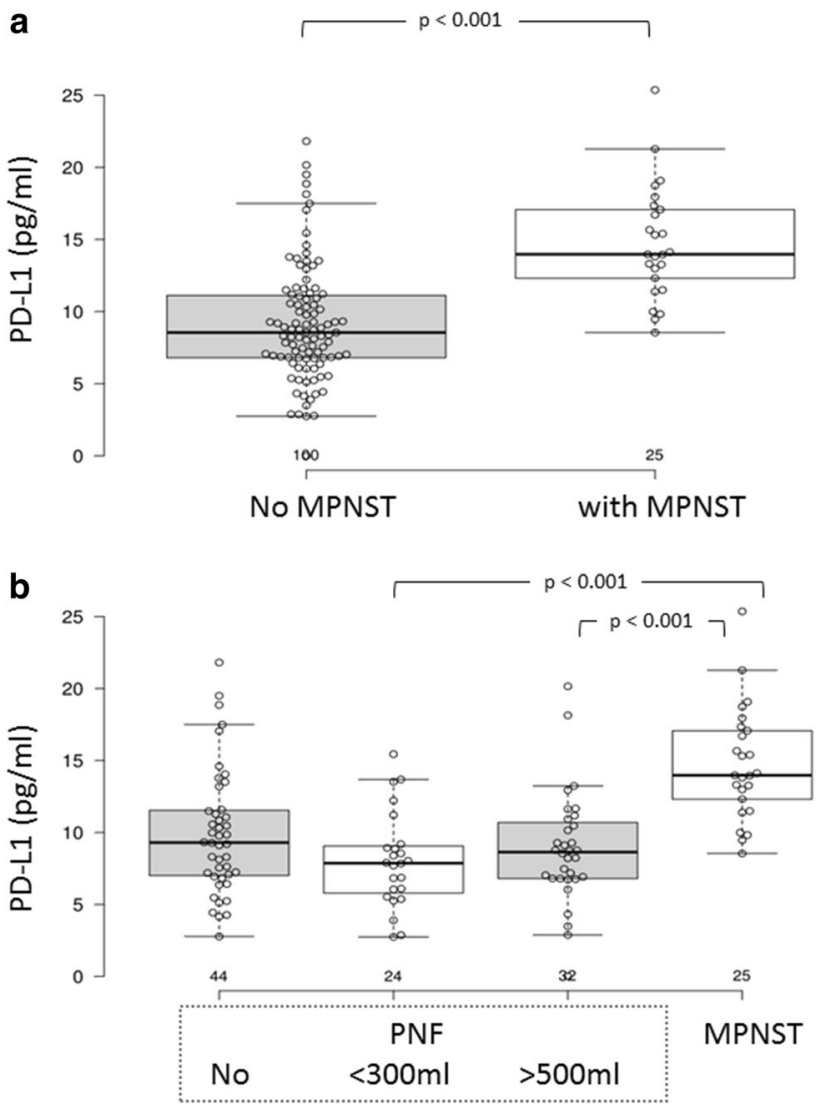

Fig. 1 Serum PD-L1 concentration in NF1 patients with and without MPNSTs. a Box plot; b distribution in patients with no MPNSTs;c distribution in patients with MPNSTs

The other tested immuno-modulators IFN- $\alpha$ and GM-CSF were below the detectable level in most of the samples and meaningful analysis therefore not possible.

None of the immuno-modulators correlated with patient age. AXL was slightly higher in male patients $(P=0.03)$. All other modulators did not differ between male and female patients (data not shown).

\section{Discussion}

The major and most important finding of the present study is the highly significantly elevated serum level of the immunomodulator PD-L1 in NF1 patients with MPNSTs. Moreover, the PD-L1 levels are particularly increased compared to patients with PNF, which are of increased risk to develop MPNSTs. Thus, PD-L1 may be a biomarker for the development of an MPNSTs in this risk group, although we do currently not know whether early stages of malignant transformation can be detected, which would require longitudinal studies. The Programmed death 1 (PD-1) inhibitory receptor is expressed by effector cytotoxic $\mathrm{T}$ cells during 

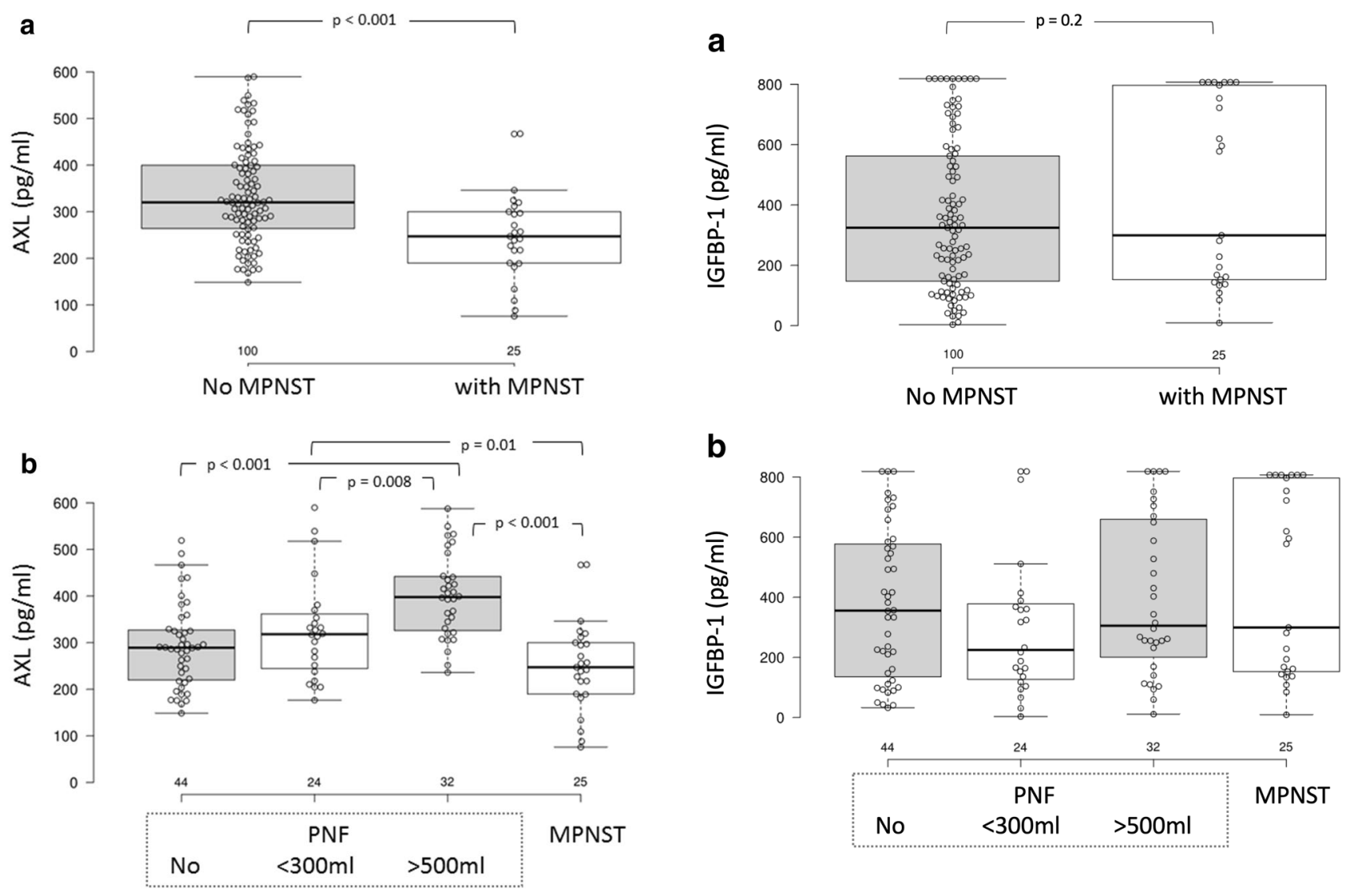

Fig. 2 Serum AXL concentration in NF1 patients with and without MPNSTs. a Box plot; $\mathbf{b}$ distribution in patients with no MPNSTs; C: distribution in patients with MPNSTs

long-term antigen exposure. Binding to its ligand, PD-L1 results in negative regulation of T cells. PD-L1 is primarily expressed in inflamed tissues and tumors. Thus, PD-L1 expression in the tumor microenvironment will reduce the T cell-based antitumor immune response [10, 23, 24]. Two anti-PD-1 monoclonal antibodies (nivolumab and pembrolizumab) [25-27] and three anti-PD-L1 monoclonal antibodies (atezolizumab, avelumab, and durvalumab) [28-30] have been approved by the US Food and Drug Administration (FDA) for treating cancers, for example, melanoma, nonsmall cell lung cancer, and renal cell carcinoma. Last year (after we completed the present study), PD-1 inhibition was reported to have achieved a complete metabolic response for a MPNSTs [31]. In addition, a trial with nivolumab and ipilimumab for rare tumors is also in progress [ClinicalTrials. gov NCT02834013]. Our finding of elevated serum levels of PD-L1 is in concordance with rational of such trials.

PD-L1 expression has been shown in circulating tumor cells, tumors and in monocytes, among others [32-39]. A recent study reported positive staining for PD-L1 in small portions ( $>5 \%$ ) of cells of a small portion (13\%) of MPNSTs and that PD-L1 expression is less profound in

Fig. 3 Serum IGFBP-1 concentration in NF1 patients with and without MPNSTs. a Box plot; $\mathbf{b}$ distribution in patients with no MPNSTs; $\mathrm{C}$ : distribution in patients with MPNSTs

benign nerve tumors including neurofibromas and schwannomas [40]. However, a more recent study did not find significant variation of the average PD-L1 staining between the MPNSTs and the benign neurofibromas [41]. In any case, we currently do not know which cells in NF1 patients with MPNSTs express and secrete PD-L1, leading to the increased serum concentration. Nevertheless, enhanced serum concentrations of PD-L1 have been indicative for attempting anti-PD-L1 is additional evidence supporting therapy [42].

We previously reported significant higher IGFBP-1 in serum of NF1 patients with MPNSTs than in NF1 patients without MPNSTs [7]. However, this finding was not confirmed in the present study including 25 MPNST patients, of which 15 showed increased IGFBP-1 serum levels ( $>30 \mathrm{ng} / \mathrm{ml}$ ). However, the MPNST cohort could be divided in two subgroups, one of which showed high IGFBP-1 serum levels. The phenotypic nature distinguishing these subgroups could not be identified, but is not due to variable PNF tumor burden. Our previous data of a reduced level of terminally differentiated CD8(+)/ CD57(+) and CD27(-) T cell fractions in NF1 patients with high benign internal tumor load may indicate a 


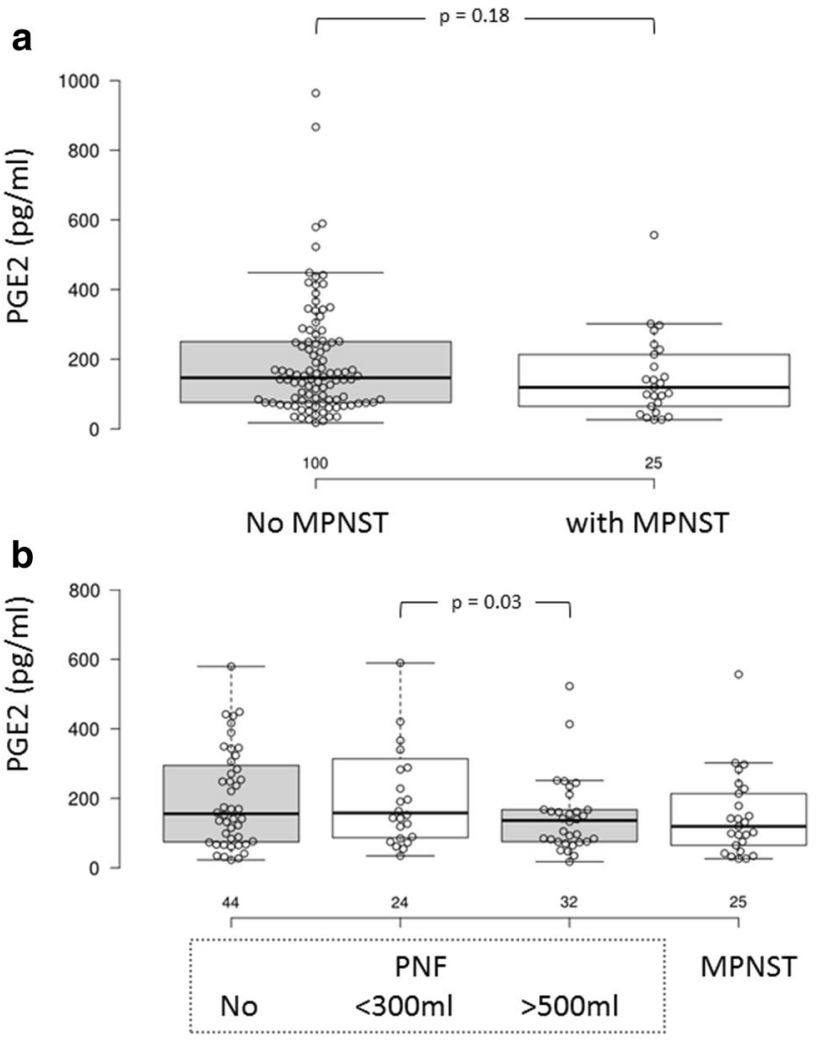

Fig. 4 Serum PGE2 concentration in NF1 patients with and without MPNSTs. a Box plot; b distribution in patients with no MPNSTs; C: distribution in patients with MPNSTs

reduced immune capacity already at the benign state, which is further exacerbated by the high PD L1 levels at the malignant state.

An unexpected finding was the significantly lowered serum level of another immuno-modulator AXL. Previous data indicated an increase in soluble AXL in patients with PNF. Our data confirmed this, and in addition, we could show that AXL serum levels are associated with internal tumor load.

Although the present study is providing strong evidence of a role for PD L1 in MPNST progression, it does not provide longitudinal and follow-up information. Future studies performed in a systematic manner to obtain samples before and after MPNST surgery, at multiple time points and longitudinal clinical assessment accompanied by immunohistochemical studies of the tumor tissue are needed to define the specific role of PD L1 for MPNST pathology. Since MPNSTs are rare tumors, multiple clinics and centers need to collaborate to enable reasonable number of cases, data and specimens.

In conclusion, the immuno-modulator PD-L1 is upregulated in MPNST patients which may provide a biomarker for early detection of malignant transformation in NF1 patients and may be a molecular target for developing new therapies.
Acknowledgements Open Access funding provided by Projekt DEAL. We would like to thank our patients for their valuable cooperation in this study.

Author contributions SF contributed to acquisition, analysis, or interpretation of data, drafting of the manuscript, and critical revision of the manuscript for important intellectual content. LK contributed to drafting of the manuscript and statistical analysis. S-JP contributed to acquisition, analysis or interpretation of data, and critical revision of the manuscript for important intellectual content. Acquisition and analysis or interpretation of data were done by S-JO. Acquisition, analysis or interpretation of data, and statistical analysis were done by NM. V-FM contributed to study concept and design, critical revision of the manuscript for important intellectual content, study outline, and supervision. AK contributed to study concept and design, acquisition, analysis or interpretation of data, critical revision of the manuscript for important intellectual content, study outline, and supervision.

Funding The work was supported by a grant from the Deutsche Krebshilfe (110588).

\section{Compliance with ethical standards}

Conflict of interest The authors declare that they have no conflict of interest.

Ethical approval The study was approved by the internal review board (Ethics Committee of the Ärztekammer Hamburg Number OB-089/04) and performed in full accordance with the Declaration of Helsinki.

Informed consent Written informed consent was obtained from all study participants.

Open Access This article is licensed under a Creative Commons Attribution 4.0 International License, which permits use, sharing, adaptation, distribution and reproduction in any medium or format, as long as you give appropriate credit to the original author(s) and the source, provide a link to the Creative Commons licence, and indicate if changes were made. The images or other third party material in this article are included in the article's Creative Commons licence, unless indicated otherwise in a credit line to the material. If material is not included in the article's Creative Commons licence and your intended use is not permitted by statutory regulation or exceeds the permitted use, you will need to obtain permission directly from the copyright holder. To view a copy of this licence, visit http://creativecommons.org/licenses/by/4.0/.

\section{References}

1. Brems H, Beert E, de Ravel T et al (2009) Mechanisms in the pathogenesis of malignant tumours in neurofibromatosis type 1. Lancet Oncol 10(5):508-515. https://doi.org/10.1016/S1470 -2045(09)70033-6

2. Evans DGR, Baser ME, McGaughran J et al (2002) Malignant peripheral nerve sheath tumours in neurofibromatosis 1 . J Med Genet 39(5):311-314. https://doi.org/10.1136/jmg.39.5.311

3. Upadhyaya MCDN (ed) (1998) Neurofibromatosis type 1: from genotype to phenotype. BIOS Scientific, Oxford

4. Ducatman BS, Scheithauer BW, Piepgras DG et al (1986) Malignant peripheral nerve sheath tumors. A clinicopathologic study of 120 cases. Cancer 57(10):2006-2021. https://doi. org/10.1002/1097-0142(19860515)57:10\%3c2006:aid-cncr2 $820571022 \% 3 \mathrm{e} 3.0 \cdot \mathrm{co} ; 2-6$ 
5. Grobmyer SR, Reith JD, Shahlaee A et al (2008) Malignant peripheral nerve sheath tumor: molecular pathogenesis and current management considerations. J Surg Oncol 97(4):340-349. https://doi.org/10.1002/jso.20971

6. Williams VC, Lucas J, Babcock MA et al (2009) Neurofibromatosis type 1 revisited. Pediatrics 123(1):124-133. https://doi. org/10.1542/peds.2007-3204

7. Park S-J, Sawitzki B, Kluwe L et al (2013) Serum biomarkers for neurofibromatosis type 1 and early detection of malignant peripheral nerve-sheath tumors. BMC Med 11:109. https://doi. org/10.1186/1741-7015-11-109

8. Farschtschi S, Park S-J, Sawitzki B et al (2016) Effector T cell subclasses associate with tumor burden in neurofibromatosis type 1 patients. Cancer Immunol Immunother 65(9):1113-1121. https:// doi.org/10.1007/s00262-016-1871-0

9. Nishimura H, Honjo T (2001) PD-1: an inhibitory immunoreceptor involved in peripheral tolerance. Trends Immunol 22(5):265-268

10. Turchinovich G, Ganter S, Bärenwaldt A et al (2018) NKp46 calibrates tumoricidal potential of type 1 innate lymphocytes by regulating TRAIL expression. J Immunol 200(11):3762-3768. https://doi. org/10.4049/jimmunol.1701333

11. Gao Y, Souza-Fonseca-Guimaraes F, Bald T et al (2017) Tumor immunoevasion by the conversion of effector NK cells into type 1 innate lymphoid cells. Nat Immunol 18(9):1004-1015. https://doi. org/10.1038/ni.3800

12. Ribas A (2012) Tumor immunotherapy directed at PD-1. N Engl J Med 366(26):2517-2519. https://doi.org/10.1056/NEJMe1205943

13. Lang D, Horner A, Brehm E et al (2019) Early serum tumor marker dynamics predict progression-free and overall survival in single PD-1/PD-L1 inhibitor treated advanced NSCLC-A retrospective cohort study. Lung Cancer 134:59-65. https://doi.org/10.1016/j. lungcan.2019.05.033

14. Wu X, Liu X, Koul S et al (2014) AXL kinase as a novel target for cancer therapy. Oncotarget 5(20):9546-9563. https://doi. org/10.18632/oncotarget.2542

15. Akslen LWR, Akslen LA, Watnick RS (eds) (2017) Biomarkers of the tumor microenvironment: basic studies and practical applications. Springer, Cham

16. Johansson G, Peng P-C, Huang P-Y et al (2014) Soluble AXL: a possible circulating biomarker for neurofibromatosis type 1 related tumor burden. PLoS ONE 9(12):e115916. https://doi.org/10.1371/ journal.pone. 0115916

17. Kirkwood JM, Butterfield LH, Tarhini AA et al (2012) Immunotherapy of cancer in 2012. CA Cancer J Clin 62(5):309-335. https ://doi.org/10.3322/caac.20132

18. Hong I-S (2016) Stimulatory versus suppressive effects of GM$\mathrm{CSF}$ on tumor progression in multiple cancer types. Exp Mol Med 48(7):e242. https://doi.org/10.1038/emm.2016.64

19. Kalinski $\mathrm{P}$ (2012) Regulation of immune responses by prostaglandin E2. J Immunol 188(1):21-28. https://doi.org/10.4049/jimmu nol.1101029

20. Ferner RE, Gutmann DH (2013) Neurofibromatosis type 1 (NF1): diagnosis and management. Handb Clin Neurol 115:939-955. https ://doi.org/10.1016/B978-0-444-52902-2.00053-9

21. Plotkin SR, Bredella MA, Cai W et al (2012) Quantitative assessment of whole-body tumor burden in adult patients with neurofibromatosis. PLoS ONE 7(4):e35711. https://doi.org/10.1371/journ al.pone.0035711

22. Mautner V-F, Asuagbor FA, Dombi E et al (2008) Assessment of benign tumor burden by whole-body MRI in patients with neurofibromatosis 1. Neuro Oncol 10(4):593-598. https://doi. org/10.1215/15228517-2008-011

23. Mallett G, Laurence A, Amarnath S (2019) Programmed cell death-1 receptor (PD-1)-mediated regulation of innate lymphoid cells. Int J Mol Sci 20(11):2836. https://doi.org/10.3390/ijms20112836
24. Salimi M, Wang R, Yao X et al (2018) Activated innate lymphoid cell populations accumulate in human tumour tissues. BMC Cancer 18(1):341. https://doi.org/10.1186/s12885-018-4262-4

25. Hamid O, Robert C, Daud A et al (2013) Safety and tumor responses with lambrolizumab (anti-PD-1) in melanoma. N Engl J Med 369(2):134-144. https://doi.org/10.1056/NEJMoa1305133

26. Wolchok JD, Kluger H, Callahan MK et al (2013) Nivolumab plus ipilimumab in advanced melanoma. N Engl J Med 369(2):122-133. https://doi.org/10.1056/NEJMoa1302369

27. Edahiro R, Kanazu M, Kurebe $H$ et al (2019) Clinical outcomes in non-small cell lung cancer patients with an ultra-high expression of programmed death ligand-1 treated using pembrolizumab as a firstline therapy: a retrospective multicenter cohort study in Japan. PLoS ONE 14(7):e0220570. https://doi.org/10.1371/journal.pone.0220570

28. Motzer RJ, Penkov K, Haanen J et al (2019) Avelumab plus axitinib versus sunitinib for advanced renal-cell carcinoma. N Engl J Med 380(12):1103-1115. https://doi.org/10.1056/NEJMoa1816047

29. Sullivan RJ, Hamid O, Gonzalez R et al (2019) Atezolizumab plus cobimetinib and vemurafenib in BRAF-mutated melanoma patients. Nat Med 25(6):929-935. https://doi.org/10.1038/s4159 1-019-0474-7

30. Antonia SJ (2019) Durvalumab after chemoradiotherapy in stage III non-small-cell lung cancer. Reply N Engl J Med 380(10):990. https ://doi.org/10.1056/NEJMc1900407

31. Davis LE, Nicholls LA, Babiker HM et al (2019) PD-1 inhibition achieves a complete metabolic response in a patient with malignant peripheral nerve sheath tumor. Cancer Immunol Res 7(9):13961400. https://doi.org/10.1158/2326-6066

32. Huang W, Ran R, Shao B et al (2019) Prognostic and clinicopathological value of PD-L1 expression in primary breast cancer: a metaanalysis. Breast Cancer Res Treat. https://doi.org/10.1007/s1054 9-019-05371-0

33. Mazel M, Jacot W, Pantel K et al (2015) Frequent expression of PD-L1 on circulating breast cancer cells. Mol Oncol 9(9):17731782. https://doi.org/10.1016/j.molonc.2015.05.009

34. Nicolazzo C, Raimondi C, Mancini M et al (2016) Monitoring PD-L1 positive circulating tumor cells in non-small cell lung cancer patients treated with the PD-1 inhibitor Nivolumab. Sci Rep 6:31726. https://doi.org/10.1038/srep31726

35. Oliveira-Costa JP, de Carvalho AF, da Silveira GG et al (2015) Gene expression patterns through oral squamous cell carcinoma development: PD-L1 expression in primary tumor and circulating tumor cells. Oncotarget 6(25):20902-20920. https://doi.org/10.18632/ oncotarget.3939

36. Rekoske BT, Olson BM, McNeel DG (2016) Antitumor vaccination of prostate cancer patients elicits PD-1/PD-L1 regulated antigenspecific immune responses. Oncoimmunology 5(6):e1165377. https ://doi.org/10.1080/2162402X.2016.1165377

37. Schehr JL, Schultz ZD, Warrick JW et al (2016) High specificity in circulating tumor cell identification is required for accurate evaluation of programmed death-ligand 1. PLoS ONE 11(7):e0159397. https://doi.org/10.1371/journal.pone.0159397

38. Satelli A, Batth IS, Brownlee Z et al (2016) Potential role of nuclear PD-L1 expression in cell-surface vimentin positive circulating tumor cells as a prognostic marker in cancer patients. Sci Rep 6:28910. https://doi.org/10.1038/srep28910

39. Bianchini M, Duchêne J, Santovito D et al (2019) PD-L1 expression on nonclassical monocytes reveals their origin and immunoregulatory function. Sci Immunol. https://doi.org/10.1126/sciimmunol .aar3054

40. Shurell E, Singh AS, Crompton JG et al (2016) Characterizing the immune microenvironment of malignant peripheral nerve sheath tumor by PD-L1 expression and presence of CD8+ tumor infiltrating lymphocytes. Oncotarget 7(39):64300-64308. https://doi. org/10.18632/oncotarget.11734 
41. Haworth KB, Arnold MA, Pierson CR et al (2017) Immune profiling of NF1-associated tumors reveals histologic subtype distinctions and heterogeneity: implications for immunotherapy. Oncotarget 8(47):82037-82048. https://doi.org/10.18632/oncotarget.18301

42. Taube JM (2014) Unleashing the immune system: PD-1 and PD-Ls in the pre-treatment tumor microenvironment and correlation with response to PD-1/PD-L1 blockade. Oncoimmunology 3(11):e963413. https://doi.org/10.4161/21624011.2014.963413

Publisher's Note Springer Nature remains neutral with regard to jurisdictional claims in published maps and institutional affiliations. 